\title{
A Methodology for Integrated System Identification, PID Controller Tuning and Noncausal Feedforward Control Design *
}

\author{
Claudio Carnevale* Aurelio Piazzi ${ }^{* *}$ Antonio Visioli* \\ * Dipartimento di Elettronica per l'Automazione, University of Brescia, \\ Italy,e-mail: \{claudio.carnevale,antonio.visioli\}@ing.unibs.it \\ ** Dipartimento di Ingegneria dell'Informazione, University of Parma, \\ Italy, e-mail: aurelio.piazzi@unipr.it
}

\begin{abstract}
In this paper we propose a systematic methodology that integrates the three main phases of the design of an industrial control system, namely, the identification phase, the tuning of the (PID) controller and the design of a (noncausal) feedforward action. In particular, the tuning of the controller is based on frequency loop shaping where the modelling uncertainty is explicitly considered and the noncausal feedforward command input synthesis is performed by applying a stable input-output inversion procedure. In this context, a parameter that allows to handle the trade-off between aggressiveness and robustness (and control effort) is given to the user. Simulation and experimental results show the effectiveness of the methodology.
\end{abstract}

\section{INTRODUCTION}

It is well-known that Proportional-Integral-Derivative (PID) controllers are the most adopted controllers in industrial settings, because they are capable to provide a satisfactory performance for a wide range of processes despite their simplicity. Actually, many tuning rules have been devised in the last sixty years in order to make their application easier (O'Dwyer (2006)). Many tuning rules are based on the assumption that a first-order-plus-deadtime (FOPDT) model of the process is available (if the process is self-regulating). Many techniques have been proposed for estimating a FOPDT model of the process based on a simple (open-loop or closed-loop) experiment. Each of them has its pros and cons (Visioli (2006)) and therefore it plays a major role in the overall control system design, but in spite of this, the identification phase is usually not related to the selected tuning rule (Leva (2005)). A notable exception in this context is the methodology proposed in (Grassi et al. (2001)), where a frequency loop-shaping approach is exploited.

From another point of view, it is also recognized that the performance of a PID control loop is determined also by the suitable implementation of those functionalities that have to (or can) be added to the basic PID control law in order to deal with practical issues (Visioli (2006)). In this context a particular attention has been paid by researchers to the synthesis of a suitable feedforward control action in order to improve the set-point following performance, especially when the tuning of the PID parameters is devoted to the load disturbance rejection performance (see, for example, (Wallen and Åström (2002); Visioli (2004))). In particular, a noncausal approach has been proposed in ( $\mathrm{Pi}$ azzi and Visioli (2006b)). It consists in applying a suitable command signal to the closed-loop control system in order

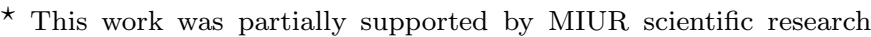
funds. to achieve a desired transient response when the process output is required to assume a new value. This command signal is determined by means of a stable input-output inversion procedure for which a closed-form solution has been determined. The desired output transition time is employed as a parameter that handles the trade-off between aggressiveness and robustness (and control effort), which is desirable in practical cases in order to address the control specifications of a given application. In any case, being a feedforward approach, the inversion technique obviously relies heavily on the accuracy of the estimated process model. Although it has been shown that the noncausal approach is effective with different identification procedures and different values of the PID parameters, in the current literature the feedforward control design is independent from the identification strategy and from the tuning of the PID controller.

Thus, in this paper we present a methodology that integrates the identification technique, the design of the PID feedback controller and the design of the noncausal feedforward control action. In particular, a prediction error/maximum likelihood method is applied to input/output data collected by applying a generalized binary noise $(\mathrm{GBN})$ signal to the process, in order to estimate a FOPDT model of the (self-regulating) process and to estimate the uncertainty bound. Then, the PID controller parameters are determined by frequency loop shaping, where the target loop shape is determined in order to achieve a high bandwidth of the system by guaranteeing at the same time the robust stability of the system. Further, the target loop shape is selected in such a way that the sensitivity function (namely, the effects of the model uncertainty) is decreased as much as possible in a wide range of (low) frequencies. Then, the command signal to be applied to the closed-loop system is determined in such a way that its frequency content is in that range, thus increasing the effectiveness of its application (Devasia (2002)). 


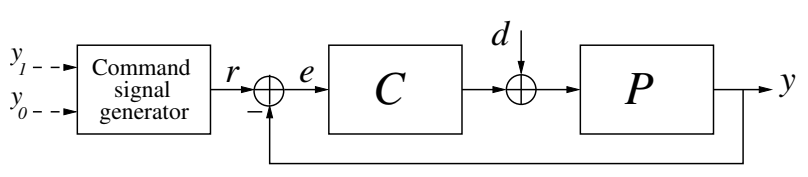

Fig. 1. The considered control scheme.

The paper is organized as follows. The overall methodology (control architecture, identification technique, PID controller tuning and feedforward control design) is explained in Section 2. Simulation results are presented in Section 3. Experimental results related to a level control problem are shown in Section 4. Conclusions are given in Section 5.

\section{METHODOLOGY}

\subsection{Control scheme}

We consider the unity-feedback control loop shown in Figure 1, where the process to be controlled (assumed to be self-regulating) is modelled as a FOPDT transfer function, i.e.:

$$
P(s)=\frac{K}{T s+1} e^{-L s} .
$$

This is a typical choice in industrial practice, since this model can describe well the dynamics of many industrial processes. Further, a FOPDT model allows to exploit an analytical solution of the stable input-output inversion procedure (Piazzi and Visioli (2006b)) (see subsection 2.4). The (output-filtered) PID feedback controller transfer function is denoted as follows:

$$
C(s)=K_{p}\left(1+\frac{1}{T_{i} s}+T_{d} s\right) \frac{1}{T_{f} s+1}
$$

where $K_{p}$ is the proportional gain, $T_{i}$ is the integral time constant, $T_{d}$ is the derivative time constant and $T_{f}$ is the time constant of a first- order filter that makes the transfer function proper. The value of $T_{f}$ can be selected, once the other parameters are determined, such that the filter dynamics does not influence the dynamics of the PID controller and the effects of the measurement noise are reduced as much as possible.

The signal $r(t)$, to be applied to the closed-loop system when a process output transition from a previous value $y_{0}$ to a new value $y_{1}$ is required, is determined by a command signal generator that implements a stable input-output inversion procedure.

\subsection{Identification}

In literature, several identification procedures have been proposed and applied (Ljung (1999)). For the purposes of the methodology proposed in this paper, obtaining a good FOPDT model and a good description of the model uncertainty is essential. It is well-known that the selection of a good test signal plays a key role in the identification. Actually, two aspects are important in selecting the input signal for process control: the signal-to-noise ratio and the power spectrum. High value of signal-to-noise ratio could be obtained using a signal with as much signal power as possible. Unfortunately, the test signal amplitude is constrained by the physical limitations of the actuators and by the nonlinearity that could be excited in the system. For this reason, for a given power, the signal with smallest amplitude is desirable. One of the indices used to identify the best signal with respect to this property is the crest factor $C_{r}$ (Guillaume et al. (1991)), defined as:

$$
C_{r}:=\frac{\max _{n} u(n)}{\sqrt{\sum_{n=1}^{N} u(n)^{2}}}
$$

where $N$ is the number of samples of the test signal. The crest factor provides a measure of how well distributed the signal values are over the input span. A good test signal must have a small crest factor; in fact, this indicates that the samples of the signal are close to their minimum or to their maximum value. For this reason, the binary signals $\left(C_{r}=1\right)$ are normally used. A GBN signal $u(t)$ assumes two values, namely, $-a$ and $+a$, which have to be selected, as already mentioned, in order to achieve the maximum amplitude of the signal without exciting the nonlinear dynamics. At each candidate switching time $t$, the amplitude may switch according to the following rule:

$$
\begin{aligned}
& P[u(t)=-u(t-1)]=p s w \\
& P[u(t)=u(t-1)]=1-p s w
\end{aligned}
$$

where $p s w$ is the switching probability, which is determined by $p s w=\frac{T_{m i n}}{E T_{s w}}$ where $T_{\min }$ is the minimum switching time (in samples) and $E T_{s w}$ is the mean switching time. The selection of the parameters $p s w$ and $E T_{s w}$ could have a deep impact on the identification results. In this work, the minimum switching time $T_{\min }$ is set to 1 (sample), while the mean switching time $E T_{s w}$ is defined as $E T_{s w}=\frac{T_{s}}{3}(\mathrm{Zhu}(2001))$ where $T_{s}$ is the $2 \%$ open-loop step response settling time. The duration of the identification experiment has been defined as $20 T_{s}$ (Zhu (2001)). The advantage of this kind of signal is that its spectrum does not present dips (unlike Pseudo Random Binary Signals) and has flexible length, allowing a simple conversion of its duration from samples to time units.

Once the input/output data are collected, a prediction error/maximum likelihood method (Ljung (1999)) is applied to them in order to estimate a FOPDT model $P(s)$ of the process and a (high-order) nominal model $P^{n}(s)$ of order $n=\sqrt{N / 40}($ Grassi et al. (2001)).

The additive uncertainty $\Delta_{a}(j w)$ is defined as the sum of two contributions: the bias due to the higher order dynamics neglected in the model, and the variance, related to the identification dataset noise:

$$
\left|\Delta_{a}(j w)\right|=\left|P^{n}\left(e^{j w}\right)-P\left(e^{j w}\right)\right|+3 \sqrt{\frac{n}{N} \frac{\Phi_{\nu}}{\Phi_{u}}}
$$

where $\Phi_{\nu}$ and $\Phi_{u}$ denote the power spectra density of the residual (with respect to $P^{n}$ ) and of the input respectively. Once the additive uncertainty is defined, the multiplicative uncertainty can be easily obtained as:

$$
\left|\Delta_{m}(j w)\right|=\frac{\left|\Delta_{a}(j w)\right|}{\left|P\left(e^{j w}\right)\right|}
$$

The closed-loop robust stability can be evaluated by considering the small gain condition

$$
|T(j \omega)|\left|\Delta_{m}(j w)\right|<1, \quad \forall \omega \in[0,+\infty)
$$

where $T(s)$ is the complementary sensitivity transfer function

$$
T(s):=\frac{C(s) P(s)}{1+C(s) P(s)} .
$$

It is worth noting that, in principle, since the model uncertainty is only estimated through the identification 
procedure, expression (7) represents an estimate rather than a rigorous sufficient condition for robust stability. Typically, for self-regulating processes, the multiplicative uncertainty is small at low frequencies and it increases for high frequencies. Denote as $\Omega$ the unity crossover frequency, namely, the frequency where

$$
\left|\Delta_{m}(j \Omega)\right|=1 \text {. }
$$

The value of $\Omega$ will represent an upper bound for the achievable bandwidth of the control system, as will be explained hereafter.

\subsection{PID controller tuning}

The tuning of the PID controller is performed, in addition to achieving the robust stability, according to the following guidelines related to both the load disturbance rejection and the set-point following task:

(1) the bandwidth of the system should be increased as much as possible in order to increase the load disturbance rejection performance (Leva (2005));

(2) the sensitivity function

$$
S(s)=\frac{1}{1+C(s) P(s)}
$$

is decreased as much as possible in a wide range of (low) frequencies, in order to reduce the effects of the model uncertainty as much as possible and therefore to increase the effectiveness of the input-output inversion technique (Piazzi and Visioli (2006a)).

Both these specifications can be met by applying a frequency loop shaping technique where the target loop shape $\bar{L}(s)$ is selected as (Grassi et al. (2001))

$$
\bar{L}(s)=\frac{\omega_{c}}{s}
$$

where the choice of the value of the target closed-loop bandwidth $\omega_{c}$ is discussed below. Thus, once the target loop shape is defined, the PID parameters can be determined so that the open-loop transfer function is as close as possible to $L(j \omega)$ in an $\mathcal{L}_{\infty}$ sense, namely, by solving the following optimization problem:

$$
\min _{K_{p}, T_{i}, T_{d}}\|C(j \omega) P(j \omega)-\bar{L}(j \omega)\|_{\infty}, \quad \omega \in\left[0,10 \omega_{c}\right] .
$$

It is worth noting that the PID controller expression can be appropriately rewritten so that the objective function is convex (affine) in the design parameters (Grassi et al. (2001)). Further, constraining the gains of the PID controller to be positive naturally avoids right-half-plane cancellations and therefore the internal stability of the system is ensured. The optimization problem can be therefore solved by applying any standard optimization technique. As already mentioned, the value of $\omega_{c}$ has to be selected in order to obtain a closed-loop system bandwidth as high as possible. The easiest way to meet this requirement is to start with a value $\omega_{c}=\Omega$ and then decrease it until the solution of the optimization problem (12) provides a PID controller that satisfies both the nominal stability and robust stability conditions. Note that for the determination of a rational transfer function $T(s)$ a Padè approximation of the dead time $L$ can be employed.

\subsection{Determination of the command input signal}

Once the feedback PID controller has been tuned, the noncausal feedforward control action $r(t)$ can be determined by applying a stable input-output inversion procedure (Piazzi and Visioli (2006b)). Roughly speaking, the procedure consists in finding the bounded closed-loop command function $r(t)$ that causes a desired output function $y_{d}(t)$. In particular, as a desired output function that defines the transition of the process variable from a set-point value $y_{0}$ to another $y_{1}$ (to be performed in the time interval $[0, \tau]$ ) a third-order "transition" polynomial (Piazzi and Visioli (2001)) can be selected, namely, a polynomial function that satisfies boundary conditions and that is parameterized by the transition time $\tau$ :

$$
y_{d}(t ; \tau)=y_{0}+y_{1}\left(-\frac{2}{\tau^{3}} t^{3}+\frac{3}{\tau^{2}} t^{2}\right)
$$

Outside the interval $[0, \tau]$ the function $y_{d}(t ; \tau)$ is equal to $y_{0}$ for $t<0$ and equal to $y_{1}$ for $t>\tau$. In the following, without loss of generality, we assume $y_{0}=0$. It is worth stressing that expression (13) represents a monotonic function with neither undershooting nor overshooting and its use is therefore very appealing in the context of process control. Further, it guarantees that a continuous command input function results.

The closed-form expression of $r\left(t ; K, T, L, K_{p}, T_{i}, T_{d}, T_{f}, \tau\right)$, defined over the interval $(-\infty,+\infty)$, that causes the desired output $y_{d}(t ; \tau)$, can be then determined by applying to the nominal closed-loop system the stable inputoutput inversion procedure presented in (Piazzi and Visioli $(2006 \mathrm{~b}))$. Actually, from a practical point of view, since the synthesized function is defined over the interval $(-\infty,+\infty)$, it is necessary to adopt a truncated function $r_{a}(t ; \tau)$, resulting therefore in an approximate generation of the desired output $y_{d}(t ; \tau)$. In particular, a preactuation time $t_{s}$ and a postactuation time $t_{f}$ can be selected so that $r_{a}(t ; \tau)=0$ for $t<t_{s}$ and $r_{a}(t ; \tau)=y_{1}$ for $t>t_{p}$. By taking into account that the preactuation and postactuation inputs (i.e. the input defined for $t<0$ and $t>\tau$ respectively) converge exponentially to zero at time $t \rightarrow-\infty$ and to $y_{1}$ at time $t \rightarrow+\infty$, an arbitrarily precise approximation can be accomplished (Piazzi and Visioli $(2005))$. Practically, the method suggested in (Perez and Devasia (2003)) can be adopted. It consists of selecting

$$
t_{s}=-\frac{10}{D_{r h p}} \quad t_{p}=\frac{10}{D_{l h p}}
$$

where $D_{r h p}$ and $D_{l h p}$ are the minimum distance of the right and left half plane zeros of $T(s)$ respectively from the imaginary axis of the complex plane.

A nice feature of the inversion-based methodology is that the output transition time $\tau$ can be effectively employed in order to handle the trade-off between aggressiveness and robustness (and control effort). In this context it is worth relating the value of $\tau$ to the value of $\omega_{c}$ previously selected (and therefore to relate it to the multiplicative uncertainty $\Delta_{m}$ ) (Devasia (2002)). Thus, the value of $\tau$ can be found as the solution of the following optimization problem:

$$
\min \tau
$$

such that

$$
|R(\omega ; \tau)|<\varepsilon|R(0 ; \tau)|, \quad \forall \omega>\omega_{c}
$$

where $R(\omega)$ is the Fourier transform of the command signal and $\varepsilon$ is now the user-chosen parameter that handles 
the trade-off between aggressiveness and robustness (obviously, the robustness decreases when $\varepsilon$ increases). From a practical point of view this optimization problem can be solved by means of a simple bisection algorithm.

\section{SIMULATION RESULTS}

In order to illustrate the overall methodology, the following (high-order) process is simulated:

$$
\frac{10}{(s+1)^{4}(3 s+1)} e^{-2 s}
$$

We selected $a=1$ for the generation of the GBN signal to be employed for identification purposes. The process output measurement has been corrupted with white noise whose power is 0.05 . The resulting input/output data are plotted in Figure 2. By applying the prediction error/maximum likelihood method, the estimated FOPDT model results to be

$$
P(s)=\frac{10.34}{5.98 s+1} e^{-3 s} .
$$

The Bode plot of the estimated model and of the multiplicative uncertainty is shown in Figure 3. It can be seen that $\Omega=1.52 \mathrm{rad} / \mathrm{s}$. In order to achieve the robust stability, the target loop shape is selected as $\bar{L}(s)=\omega_{c} / s$ where $\omega_{c}=0.18 \mathrm{rad} / \mathrm{s}$. The PID parameters that minimize (12) are $K_{p}=0.10, T_{i}=5.94, T_{d}=0.15$. Then, $T_{f}=0.01$ has been selected. The bode plot of the closed-loop transfer function $T(s)$ together with that of $1 / \Delta_{m}$ is shown in Figure 4.

By selecting $\varepsilon=0.05$ in the inversion procedure, we obtain $\tau=56.9$. The resulting command input function for $y_{1}=1$ is shown in Figure 5. The corresponding process output, together with the resulting control variable is shown in Figure 6 where results obtained with the application of a step input are also shown for comparison. Note that the presence of a time delay in the closed-loop response when the noncausal approach is employed is due to the presence of the preactuation time interval.

From the presented example, it turns out that, despite the high-order dynamics of the process and the significant dead time, the PID tuning is satisfactory. The oscillatory set-point step response is then avoided by implementing the inversion-based procedure, which ensures a limitation of the overshoot. It is worth noting again that a more aggressive response with the inversion-based approach can be obtained by increasing the parameter $\varepsilon$. This fact can be understood better by evaluating the results obtained with $\varepsilon=0.1$ and $\varepsilon=0.15$ (corresponding to $\tau=22.2$ and $\tau=11.4$ respectively) that are shown in Figure 7 .

\section{EXPERIMENTAL RESULTS}

In order to prove the effectiveness of the devised technique in practical applications, a laboratory experimental setup (made by KentRidge Instruments) has been employed. Specifically, the apparatus consists of small perspex towertype tank (whose area is $40 \mathrm{~cm}^{2}$ ) in which a level control is implemented by means of a PC-based controller. The tank is filled with water by means of a pump whose speed is set by a DC voltage (the manipulated variable), in the range $0-5 \mathrm{~V}$, through a PWM circuit. The tank is fitted with an outlet at the base in order for the water to return
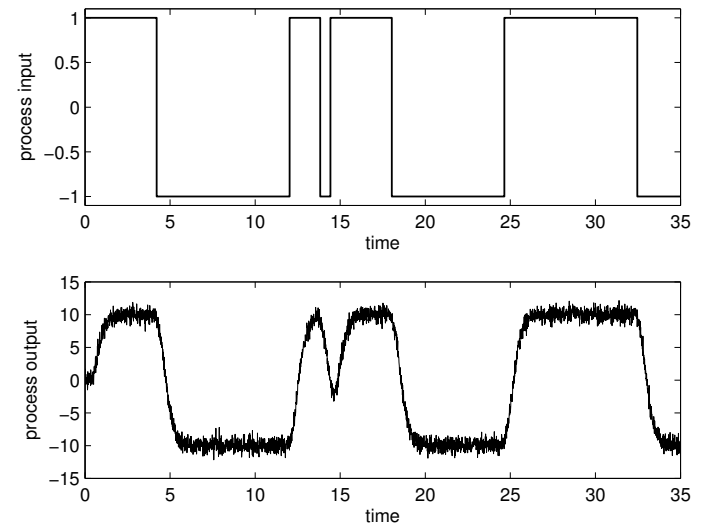

Fig. 2. Input and output data employed for identification in the simulation example.

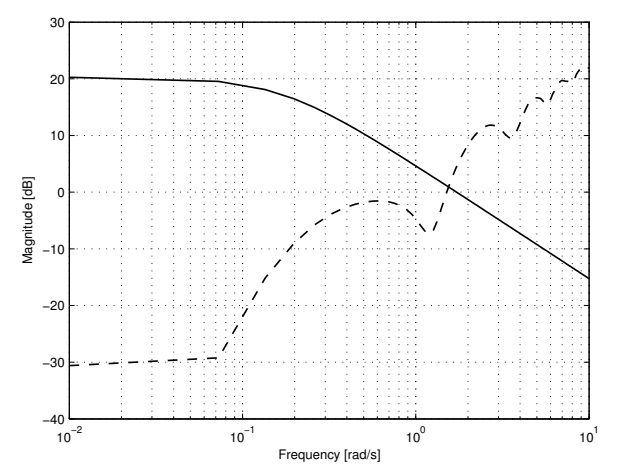

Fig. 3. Bode plot of the estimated model $P(s)$ (solid line) and of the multiplicative uncertainty $\Delta_{m}(s)$ (dashed line) for the simulation example.

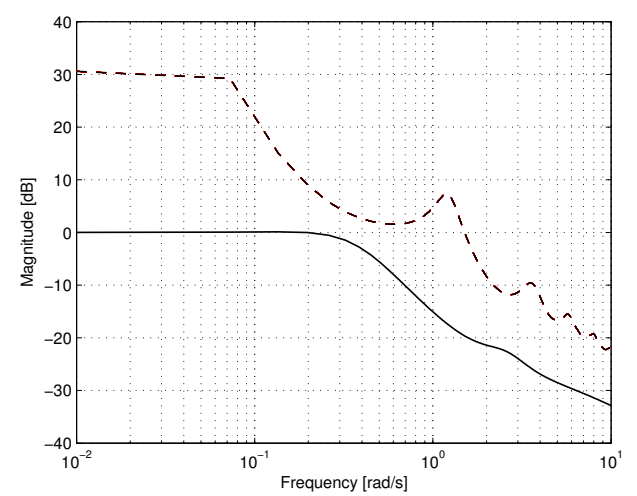

Fig. 4. Bode plot of the closed-loop system $T(s)$ (solid line) and of $1 / \Delta_{m}(s)$ (dashed line) for the simulation example.

to a reservoir. The measure of the level of the water is given by a capacitive-type probe that provides an output signal between 0 (empty tank) and $5 \mathrm{~V}$ (full tank). For the sake of simplicity, in the following the level variable will be expressed in Volts. Note that the system has nonlinear dynamics, because the flow rate out of the tank depends on the square root of the level. The control task consists in achieving a process variable transition from $y_{0}=2 \mathrm{~V}$ to $y_{1}=3 \mathrm{~V}$. 


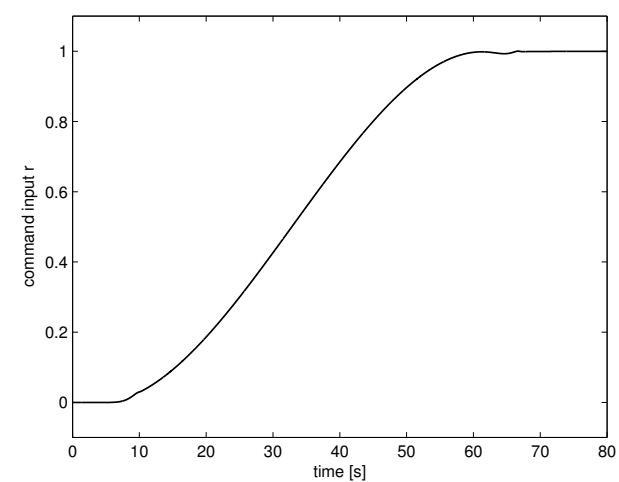

Fig. 5. Command input function $r$ for the simulation example.
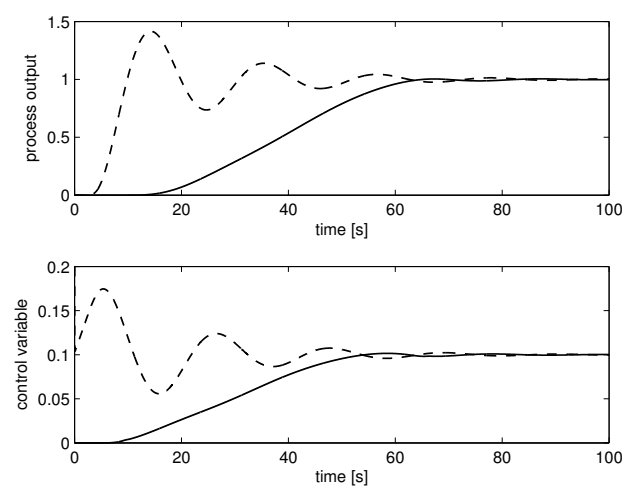

Fig. 6. Process output and control variable with the determined command input (solid line) and with a step set-point signal (dashed line) for the simulation example.
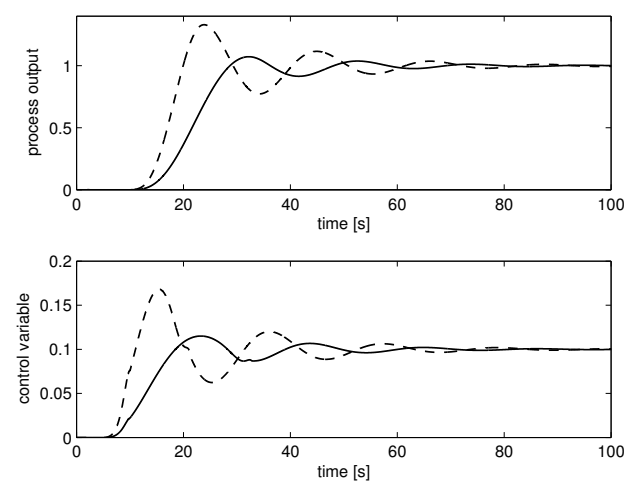

Fig. 7. Process output and control variable with $\varepsilon=0.1$ (solid line) and with $\varepsilon=0.15$ (dashed line) for the simulation example.

The lower and upper limits of the GBN signal have been selected as $2 \mathrm{~V}$ and $3 \mathrm{~V}$ respectively, in order to avoid too much exciting of the nonlinearity. The resulting input and output data for identification are shown in Figure 8. The result of the prediction error/maximum likelihood method is

$$
P(s)=\frac{1.59}{26.7 s+1} e^{-2.96 s} .
$$
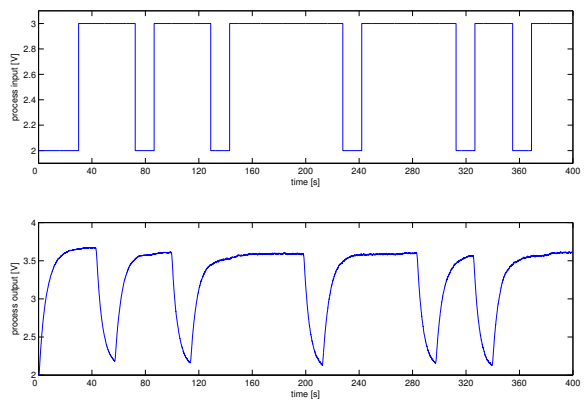

Fig. 8. Input and output data employed for identification in the level control example.

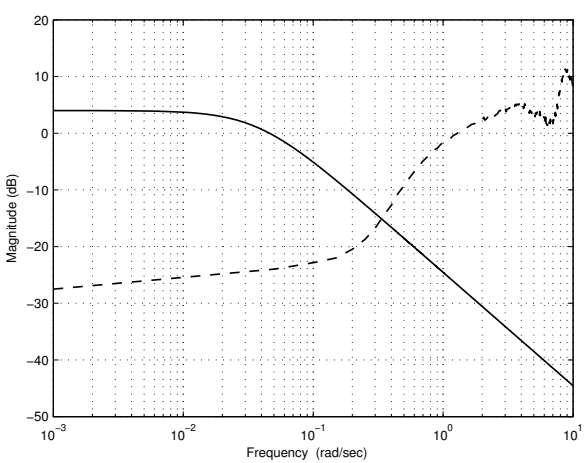

Fig. 9. Bode plot of the estimated model $P(s)$ (solid line) and of the multiplicative uncertainty $\Delta_{m}(s)$ (dashed line) for the level control example.

The Bode plot of the estimated model and of the multiplicative uncertainty is shown in Figure 9, where $\Omega=$ $1.31 \mathrm{rad} / \mathrm{s}$. The robust stability is achieved by selecting the target loop shape as $\bar{L}(s)=0.45 / s$. The PID parameters that minimize $(12)$ are found as $K_{p}=6.11, T_{i}=19.52$, $T_{d}=0.26\left(T_{f}=0.01\right)$. The Bode plot of the closed-loop transfer function $T(s)$ together with that of $1 / \Delta_{m}$ is shown in Figure 10.

Two values of $\varepsilon$ have been considered, namely, $\varepsilon=0.015$, which corresponds to $\tau=10.31 \mathrm{~s}$ and $\varepsilon=0.016$ which corresponds to $\tau=3.59 \mathrm{~s}$. The obtained inversion-based command input signals are shown in Figure 11, while the corresponding process variables and control variables are shown in Figures 12 and 13 respectively. The step response has been also plotted for comparison. Note that, disregarding the time delay due to the presence of a preactuation time interval, the use of the noncausal feedforward control action allows to decrease significantly the overshoot with respect to the use of a step signal, without increasing the rise time. Note also that the control variable exceed the actuator saturation limit of $5 \mathrm{~V}$ both for the step signal and for the inversion-based command signal when $\varepsilon=0.016$. This does not occur when $\varepsilon=0.015$, confirming that parameter $\varepsilon$ allows to handle the control effort effectively.

\section{CONCLUSIONS}

In this paper a methodology that integrates the identification technique, the feedback PID controller tuning and the (noncausal) feedforward action has been proposed. 


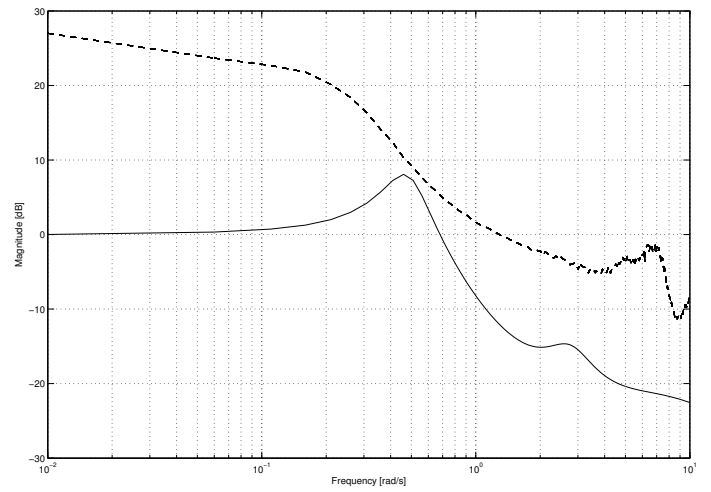

Fig. 10. Bode plot of the closed-loop system $T(s)$ (solid line) and of $1 / \Delta_{m}(s)$ (dashed line) for the simulation example.

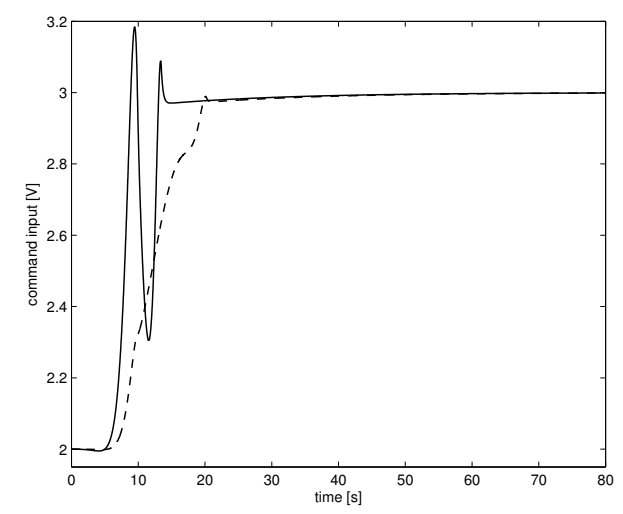

Fig. 11. Command input functions $r$ for the level control example. Solid line: $\varepsilon=0.015$; dashed line: $\varepsilon=0.016$.

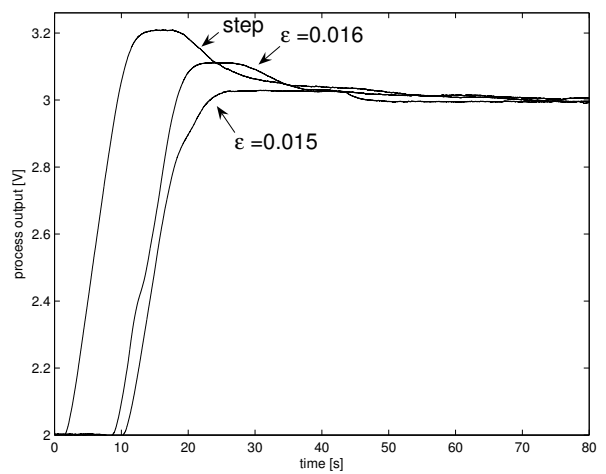

Fig. 12. Process variable for different command input signals for the level control example.

It has to be remarked that the overall procedure can be automated. Further, a user-chosen parameter allows easy handling of the trade-off between aggressiveness and robustness, which is a very desirable feature in practical cases. Simulation and experimental results have demonstrated the effectiveness of the approach even in the presence of processes with a high-order dynamics or nonlinear dynamics.

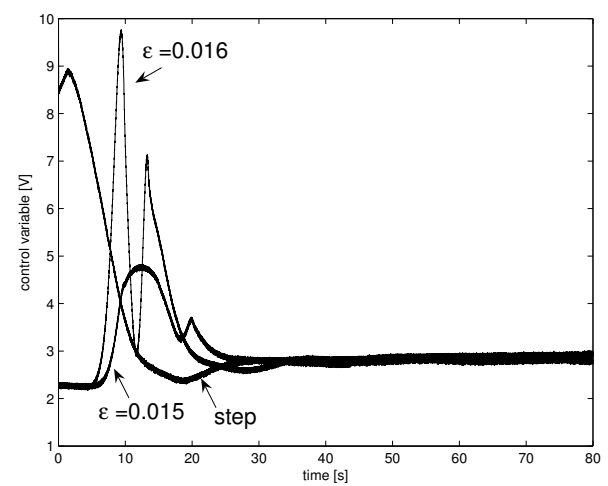

Fig. 13. Control variable for different command input signals for the level control example.

\section{REFERENCES}

S. Devasia. Should model-based inverse inputs be used as feedforward under plant uncertainties? IEEE Transactions on Automatic Control, 47(11):1865-1871, 2002.

E. Grassi, K. S. Tsakalis, S. Dash, S. V. Gaikwad, W. MacArthur, and G. Stein. Integrated system identification and PID controller tuning by frequency loopshaping. IEEE Transactions on Control Systems Technology, 9:285-294, 2001.

P. Guillaume, J. Schoukens, R. Pintelon, and I. Kollar. Crest-factor minimization using nonlinear Chebyshev approximation methods. IEEE Transactions on Instrumentation and Measurement, 40(6):982-989, 1991.

A. Leva. Autotuning process controller with improved load disturbance rejection. Journal of Process Control, 15: 223-234, 2005.

L. Ljung. System Identification: Theory for the Users. Prentice Hall, New Jersey, 1999.

A. O'Dwyer. Handbook of PI and PID Tuning Rules. Imperial College Press, 2006.

H. Perez and S. Devasia. Optimal output transitions for linear systems. Automatica, 39:181-192, 2003.

A. Piazzi and A. Visioli. Optimal noncausal set-point regulation of scalar systems. Automatica, 37(1):121-127, 2001.

A. Piazzi and A. Visioli. Using stable input-output inversion for minimum-time feedforward constrained regulation of scalar systems. Automatica, 41(2):305-313, 2005.

A. Piazzi and A. Visioli. Combining $h_{\infty}$ control and dynamic inversion for robust constrained set-point regulation. In Preprints IFAC Workshop on Control Applications of Optimisation, pages 209-214, Paris-Cachan (F), 2006a.

A. Piazzi and A. Visioli. A noncausal approach for PID control. Journal of Process Control, 16:831-843, 2006b.

A. Visioli. Practical PID Control. Springer, London (UK), 2006.

A. Visioli. A new design for a PID plus feedforward controller. Journal of Process Control, 14:455-461, 2004.

A. Wallen and K. J. Åström. Pulse-step control. In Preprints of the 15th IFAC World Congress on Automatic Control, Barcelona, E, 2002.

Y. Zhu. Multivariable System Identification for Process Control. Elsevier Science, United Kingdom, 2001. 